\title{
Difficulties in localization and treatment of insulinomas in type 1 multiple endocrine adenomatosis (MEA)
}

\author{
Peter H. Winocour ${ }^{1}$, Kieran J. Moriarty ${ }^{1}$, C. Nicholas Hales ${ }^{4}$, Judy Adams ${ }^{2}$, \\ Roy Reeve ${ }^{3}$, David Wynick ${ }^{5}$, David Allison ${ }^{6}$, Steven R. Bloom ${ }^{5}$ and \\ David C. Anderson ${ }^{1}$
}

University of Manchester Departments of ${ }^{1}$ Medicine, ${ }^{2}$ Diagnostic Radiology and ${ }^{3}$ Histopathology, Hope Hospital, Salford, ${ }^{4}$ University of Cambridge Department of Clinical Biochemistry, and University Departments of ${ }^{5}$ Medicine and ${ }^{6}$ Diagnostic Radiology, Hammersmith Hospital, London, UK

\begin{abstract}
Summary: A 15 year old girl with a family history of type 1 multiple endocrine adenomatosis presented with reversible neurological disturbances, hypoglycaemia and hyperinsulinaemia. Initial radiology was normal, but portal venous sampling suggested an insulinoma in the tail of the pancreas which was removed with conservation of the spleen. Hypoglycaemia persisted despite high doses of diazoxide and intravenous dextrose. A second laparotomy revealed a pancreatic endocrine tumour and sub-total pancreatectomy was performed. Histology revealed islet cell microadenomatosis. Hypoglycaemia persisted despite treatment with somatostatin analogues and $\mathbf{4 0 \%}$ intravenous dextrose was required to maintain normoglycaemia. A possible lesion near the splenic hilum on computed tomographic scan was reported as a splenunculus although further peripheral, hepatic and portal venous sampling suggested hepatic or systemic lesions. A positron emission scan and selective visceral angiography suggested a lesion in the left upper quadrant. Acute lactic acidosis, rhabdomyolysis and renal failure supervened. Post mortem revealed the putative 'splenunculus' to be a residual insulinoma, whilst the splenic vein was thrombosed, accounting in part for discrepant venous sampling data.

Hyperinsulinaemia in type 1 multiple endocrine adenomatosis may require more aggressive surgical and hormonal intervention than when dealing with solitary insulinomas. Insulinomas may mimic developmental abnormalities on computed tomographic scanning.
\end{abstract}

\section{Introduction}

Hyperinsulinism associated with type 1 multiple endocrine adenomatosis (MEA) is often the consequence of multiple benign insulinomas or diffuse microadenomatosis. ${ }^{1-3}$ Consequently, management strategy may be rather different than for solitary insulinomas.

\section{Case history}

A 15 year old girl, previously well, presented in August 1986 with episodes of reversible neurological disturbances. During one episode of convulsions, hypoglycaemia of $1.5 \mathrm{mmol} / \mathrm{l}$ was noted.

Correspondence: P.H. Winocour, M.D., University of Newcastle upon Tyne, Department of Medicine, The Medical School, Framlington Place, Newcastle upon Tyne, NE2 4HH, UK.

Accepted: 16 October 1991
Subsequently frequent hypoglycaemia and inappropriate hyperinsulinaemia $(245 \mathrm{pmol} / 1$, normal under $30 \mathrm{pmol} / \mathrm{l})$, elevated $\mathrm{C}$-peptide levels (1.56 pmol/1, normal under $1.5 \mathrm{pmol} / 1)$, and mild hyperchloraemic acidosis associated with elevated parathyroid hormone levels $(94 \mathrm{pg} / \mathrm{ml}$, normal under $50 \mathrm{pg} / \mathrm{ml}$ ) were recorded. There was a family history of type 1 MEA. Her father had previously had surgery for pituitary and parathyroid adenomata and gastroduodenal surgery for recurrent peptic ulceration, due to presumed gastrinoma. Two brothers had presented with peptic ulceration at the age of 16 years.

An insulinoma was suspected, but abdominal computed tomographic (CT) scan and pancreatic arteriography were essentially normal. Portal venous sampling showed an insulin gradient $(518 \mathrm{vs}$ $350 \mathrm{pmol} / \mathrm{l})$ between the tail and the head of the pancreas. At laparotomy, the tail of the pancreas was removed, although no obvious pancreatic tumour was visualized. The appearance of the 
spleen was not commented on and it was preserved. Histology of the pancreatic tail revealed diffuse islet cell hyperplasia with one markedly enlarged islet similar to those seen subsequently and regarded as microadenomata.

Hyperinsulinism and hypoglycaemia persisted post-operatively. A further abdominal CT scan was non-contributory and selective portal venous sampling showed an insulin peak in the portal vein. This was thought to represent drainage from the remaining head of pancreas. Treatment with diazoxide and chlorothiazide led to marginal improvement and the patient was transferred to our care for further management. A third abdominal CT scan did not reveal any abnormality.

In September 1987, laparotomy was performed, when a $1.5 \times 1 \times 0.5 \mathrm{~cm}$ tumour was identified in the body of the pancreas, and consequently a sub-total pancreatectomy was performed. Histology confirmed that an endocrine cell tumour had been removed, which stained weakly for insulin and did not grow in culture. No microadenomata were present although diffuse islet cell hyperplasia was noted. These islets stained positively for both insulin and glucagon with immunoperoxidase but not for somatostatin.

Post-operative hypoglycaemia continued despite the administration of intravenous $10 \%$ dextrose, which necessitated reinstitution of diazoxide. A somatostatin infusion test led to a marked suppression of insulin with a rise of blood glucose levels. However, there was little change in proinsulin levels when measured by specific immunoradiometric assay. ${ }^{4}$ Treatment with the long-acting somatostatin analogue SMS 201-995 (Sandoz Pharmaceuticals, UK) $50 \mu \mathrm{g}$ b.d. produced headaches, and only transient normoglycaemia so it was discontinued. At this time, pituitary and gastrointestinal hormone (VIP, somatostatin, glucagon, bombesin, enterolglucagon) assays were reported as normal.

Simultaneous peripheral and hepatic venous sampling showed high levels of insulin and proinsulin in branches of the hepatic vein, but peripheral levels appeared higher than central levels, after correction for differences in albumin concentration. A bronchial carcinoid secreting insulin was considered, but a thoracic CT scan showed no abnormality. The abdominal CT scan was repeated at this examination and was again reported as normal, with the exception of a putative splenunculus, which was also demonstrated on review of the previous CT scans (Figure la,b). Transhepatic portal venous sampling demonstrated peak levels of insulin and proinsulin in the region of the porta hepatis, possibly representing drainage downstream from the pancreatico-duodenal veins. Endoscopic ultrasound purported to show an intrapancreatic nodule in the head of the pancreas a

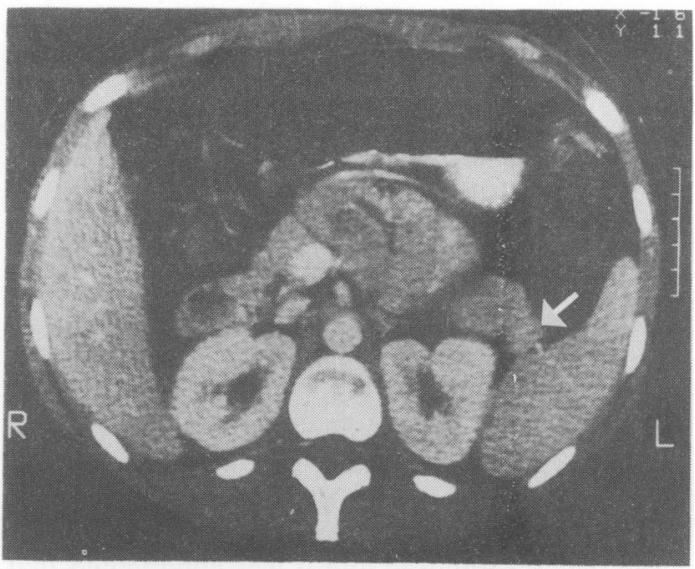

b

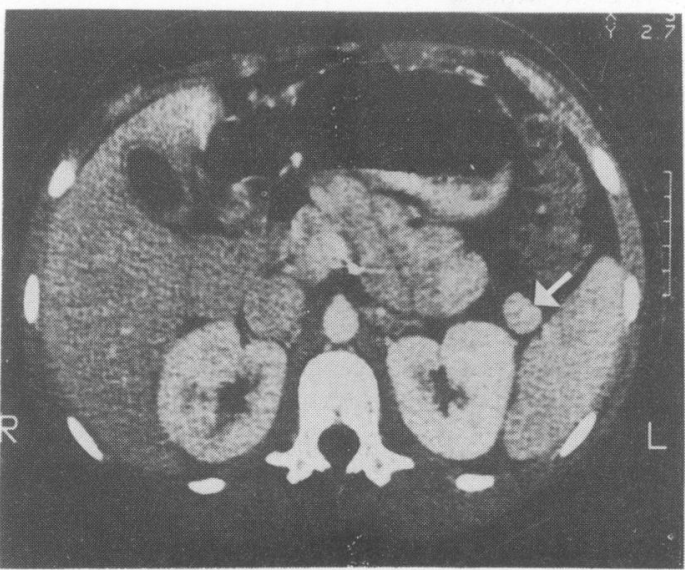

Figure 1 Abdominal CT scans in a, August 1986 (with contrast) demonstrating insulinoma (arrowed) apparently attached to normal splenic and pancreatic tissue, and b, September 1987 (with contrast) demonstrating the insulinoma (arrowed) adjacent to the spleen of similar CT scan density.

and an extrapancreatic nodule of uncertain significance near the inferior vena cava. A fifth abdominal $\mathrm{CT}$ scan provided no new information because of streak artefact from metal surgical clips degrading the image.

Hypoglycaemia continued despite intravenous dextrose and diazoxide, and a pancreaticoduodenectomy was carried out in January 1988. At laparotomy the surgeon palpated but did not clearly visualize what had previously been reported as the splencuculus. The belief that the splenunculus had been identified at surgery strengthened the view that this lesion was not an insulinoma. No tumour was seen but histology of the remnant pancreatic tissue showed multiple islet cell micro- 
adenomata which had positive immunoperoxidase staining for glucagon and insulin within some but not all of the tumour. Perioperative peripheral and portal venous sampling revealed the highest insulin levels in the portal vein and a post-operative reduction in insulin secretion was recorded on a rapid plasma insulin assay carried out at the time of surgery.

Significant post-operative hypoglycaemia recurred, which required intravenous dextrose, diazoxide and intermittent parenteral glucagon. Glucagon produced rises in blood glucose but this was associated with subsequent rebound peripheral hyperinsulinaemia and hypoglycaemia.

In March 1988, hepatic arteriography demonstrated a small lesion in the splenic hilum corresponding to the putative splenunculus. The splenic vein was thrombosed and it was commented that the drainage from this area could consequently be via collaterals into the azygos vein. Central venous sampling again showed highest insulin levels in the portal vein, and a hepatic tumour with anomalous drainage was considered. Hypoglycaemia at this time was severe enough to necessitate 8 hourly infusions of $20 \%$ dextrose in addition to $1 \mathrm{~g}$ diazoxide. Somatostatin infused at a rate of $250-500 \mu \mathrm{g} / \mathrm{h}$ effectively suppressed insulin levels and produced a slightly delayed peripheral hyperglycaemic response (Figure 2).

Positron emission tomography (PET) suggested a left upper quadrant lesion and selective visceral angiography, with injection of the remnant of the dorso-pancreatico-duodenal artery, demonstrated a tumour blush in the midline, anterior to the $\mathrm{T} 12 / \mathrm{L} 1$ disc interspace.
Unfortunately the patient's condition deterior ated. She refused to continue with oral diazoxidez which had caused severe hirsutism and fluid reten $\mathbb{\mathbb { Q }}$ tion, and became fatigued, nauseated, pyrexial, anaemic and hypokalaemic. A further abdominat CT scan (the sixth) was reported as showing? extensive fatty change in the liver and the pree sumed splenunculus was again noted at the splenio. hilum'. Severe hypoglycaemia persisted and $40 \% \frac{\infty}{-}$ intravenous glucose via a central line was required to maintain normoglycaemia. Cultures of bloow and of the central line were negative and further abdominal and pelvic CT scans unhelpful. She developed severe lactic acidosis, rhabdomyolysisw and renal failure and died on the 15 July 1988 .

Post mortem examination revealed generalize oedema, severe mediastinal and peritoneal haem orrhage, necrotizing myopathy, and a tumour ato the splenic hilum attached to normal pancreatic taito tissue. This was subsequently confirmed on his- $-\mathrm{V}$ tology to be an insulinoma. No splenunculus wase seen and there was no evidence of pituitary? parathyroid, thyroid or adrenal adenomata.

\section{Discussion}

This case raises the issue of the optimum approaghe to diagnosis and management of hyperinsu naemia associated with type $1 \mathrm{MEA}$ in a youfig patient. Many reports on the diagnosis and treatment of insulinoma ${ }^{5-7}$ refer to sporadic cases withō solitary tumours, which may on occasion metas-⿳亠丷厂 tasize, whereas it is evident from studies in type $1 \stackrel{\circ}{\circ}$ MEA that hyperinsulinism is due to benign multi- $\overrightarrow{\vec{P}}$

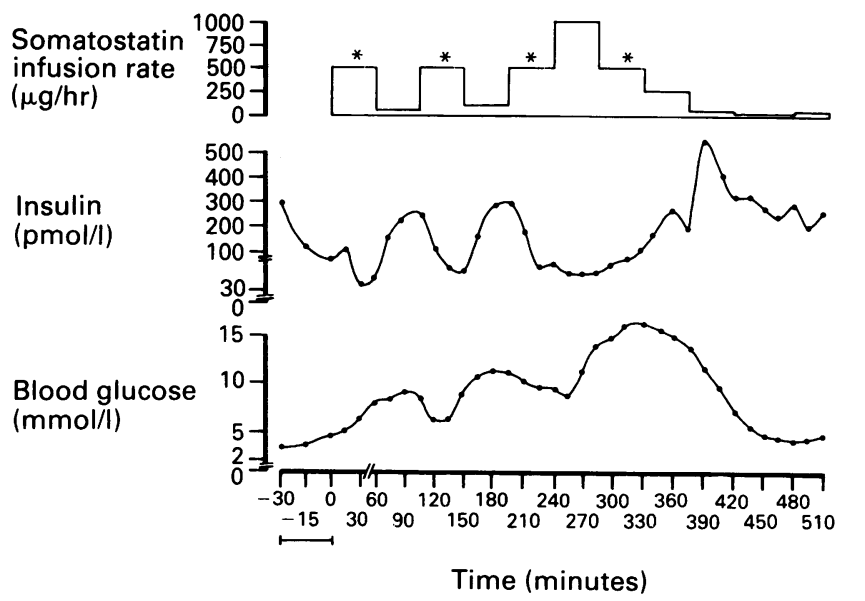

Figure 2 Peripheral insulinaemic and glycaemic responses to intravenous somatostatin at different infusion rates. $\frac{\vec{C}}{\vec{D}}$ *500 $\mu \mathrm{g} / \mathrm{h}$ considered threshold dose. Note lag period in insulin and glucose responses and evidence of breakthrough $\frac{\mathcal{P}}{\mathbb{P}}$ and rebound insulin hypersecretion. 
ple microscopic adenomatosis in up to $70 \%$ of cases. ${ }^{1-3}$ Furthermore $1 \%$ of insulinomas in type 1 MEA arise at extrapancreatic sites. ${ }^{3}$ Consequently, more radical surgery might have been more appropriate at the initial laporotomy in this case, particularly since much of the later management was made more difficult by the effects of previous surgery. Although pancreatectomy and splenectomy might have proved curative because of the location of the insulinoma, no mention is made of this in reviews of surgical treatment of type 1 MEA. $^{8}$

In addition, other features of this case merit discussion:

Imaging of lesions It was ironic that the residual insulinoma which was implicated in the patient's eventual demise had been visualized on at least 4 abdominal CT scans, and was also located by hepatic arteriography. However, the appearance was consistently reported as a splenunculus despite considerable discussion on this point, and this certainly had a bearing on further surgical management. Although the vascularity and the density of the enhanced CT image were not thought to be typical of aberrant pancreatic tissue, similar density of splenic and insulinoma tissue on CT scan following injection of contrast is recognized and was misleading in this case. However, it is reasonable to suggest in retrospect, whether or not a splenunculus had been present, that the nesidioblastosis/apudomas of type 1 MEA could be expected to develop in developmental anomalies such as a splenunculus. The use of endoscopic ultrasound in the current case proved misleading in the localization of the tumour. Such difficulties in localization of multiple endocrine adenomata have previously been recognized in at least one report. ${ }^{9}$

Blood sampling for insulin Although it is said that transhepatic venous sampling may give the highest chance of accurate localization, ${ }^{1}$ peripheral and central insulin and proinsulin levels suggested, at various times, hepatic 'metastases', a bronchial carcinoid, or a continued source of insulin/proinsulin secretion in the distribution of the pancreatico-duodenal circulation. In our patient, the anomalous venous drainage from the tumour at the splenic hilum, secondary to thrombosis of the splenic vein or to previous surgery, contributed to these discrepancies. It should be emphasized, however, that hepatic arteriography demonstrated this feature and the sampling data might have been interpreted differently had this information been available at the time. Episodic hormonal secretion from the tumour may also have contributed to some of the discrepancies.

Hormonal secretion and responsiveness Although the resection of the residual tumour at the splenic hilum might have been curative, there is considerable support for the use of somatostatin analogues as effective treatment for metastatic islet cell tumours. ${ }^{7,10-12}$ The islet cell tumour(s) in the current case remained hormonally sensitive, not only to native infused somatostatin but also to glucagon, although it was predominantly elevated insulin levels, rather than proinsulin, that responded. Furthermore, despite the suggestion that the dose-response curve of islet cell tumours to somatostatin or its longer-acting analogue is especially shallow, ${ }^{11,12}$ we were able to demonstrate a threshold level of somatostatin infusion on insulin and blood glucose levels $(250-500 \mu \mathrm{g} / \mathrm{h})$. The response to the somatostatin analogue was rather disappointing, although it should be noted that low doses only were used owing to presumptive side effects and the hope at the time that curative surgical treatment was imminent. On reflection, there would have been a case for the reintroduction of higher doses of the somatostatin analogue, perhaps in conjunction with chemotherapy, but the patient's condition deteriorated when this option was being considered.

Longer term management of persistent hypoglycaemia The hypoglycaemia appeared more aggressive after each diagnostic and therapeutic intervention, and this necessitated administration of high concentrations of intravenous glucose. This probably led to fatty infiltration of the liver and fluid retention, which together with the lack of absorption of dietary protein and fat, may have contributed to the metabolic acidosis and myopathy which led to renal failure and death.

It is possible that earlier aggressive hormonal treatment and chemotherapy might have led to lower glucose requirements which might have helped protect the liver from severe fatty infiltration. ${ }^{10-12}$

In conclusion, insulinomas in type 1 MEA are frequently multiple, and careful, extensive preoperative localization of tumours is necessary. However, diagnostic techniques for the localization of insulinomas must be interpreted with great caution. Initial radical appropriate surgical treatment may be curative and hormonal treatment can be beneficial, while unopposed high concentrations of intravenous dextrose and nutritional imbalance may be particularly dangerous. 


\section{References}

1. Le Quesne, L.P. \& Daggett, P.R. Insulin tumours of the pancreas. In: Johnston, I.D.A. \& Thompson, N.W. (eds) Endocrine Surgery. Butterworth, London, 1983, pp. $104-124$.

2. Vance, J.E., Stoll, R.W., Kitabachi, A.E. et al. Nesidioblastosis in familial endocrine adenomatosis. JAMA 1969, 207: 1679.

3. Lips, C.J., Vasen, H.F. \& Lamers, C.B. Multiple endocrine neoplasia syndromes. Crit Rev Oncol Haematol 1984, 2: 117-184.

4. Sobey, W.J., Beer, S.F., Carrington, C.A. et al. Sensitive and specific two-site immunoradiometric assays for human insulin, proinsulin, 65-66 split and 32-33 split proinsulins. Biochem J 1989, 260: 535-541.

5. Broder, L.E. \& Carter, S.K. Pancreatic islet cell carcinoma Clinical features of 52 patients. Ann Int Med 1973, 79, $101-107$.

6. Stefanini, P., Carboni, M., Patrassi, N. et al. Beta-islet cell tumours of the pancreas: Results on a study of 1067 cases. Surgery 1974, 75: 597-609.
7. Kvols, K.L., Buck, M., Moertel, C.G. et al. Treatment of metastatic islet cell carcinoma with a somatostatin analogue (SMS-201-995). Ann Intern Med 1987, 107: 162-168.

8. Thompson, N.W. Surgical considerations in the MEA type $1 \overrightarrow{\vec{B}}$ syndrome. In: Johnston, I.D.A. \& Thompson, N.W. (eds) Endocrine Surgery. Butterworths, London, 1983, pp. $151-161$.

9. Doppman, J.L. Multiple endocrine syndromes - a nightmare $\frac{\overline{\bar{D}}}{\bar{D}}$ for the endocrinologic radiologist. Sem Roentg 1985, 20: $\overparen{\Phi}$ $7-16$.

10. Lang, R.G., Barnes, A.J., Adrian, T.E. et al. Suppression of صి pancreatic endocrine tumour secretion by long-acting somatostatin analogue. Lancet 1979, 2: 764-767.

11. Chung, J.H., Williams, S.J. \& Bloom, S.R. Chronic treatment of gut hormone producing tumours (apudomas) with a $\vec{\omega}$ long-acting somatostatin analogue. Dig Dis Sci 1983, 28: ڤ $577-584$.

12. Bonfils, S. New somatostatin molecule for management of endocrine tumours. Gut 1985, 26: 433-437. 